\title{
Univariate equation-based SHE-PWM for multilevel inverter
}

\author{
PIYUSH L KAMANI (1) and MAHMADASRAF A MULLA* (1)
}

Department of Electrical Engineering, Sardar Vallabhbhai National Institute of Technology, Surat 395009, India

e-mail: kamanipiyush03@gmail.com; mamulla@ieee.org

MS received 8 October 2018; revised 7 February 2019; accepted 19 February 2019; published online 20 April 2019

\begin{abstract}
This article presents a selective harmonic elimination-pulse-width modulation (SHE-PWM) method for cascaded H-bridge multilevel inverters. The concept of volt-second area balancing is applied to estimate the voltage ratings of the dc sources, which provides different voltage ratings of each dc source. The control of output voltage is achieved by varying switching notch created at the centre of each level. This method calculates switching angles in real time easily owing to the usage of univariate equations. A comparison study shows that the proposed method eliminates more harmonics compared with the conventional SHE-PWM methods. Simulation and experimental studies are conducted to validate the performance of the proposed SHE-PWM method.
\end{abstract}

Keywords. Multilevel inverter; pulse-width modulation; selective harmonic elimination.

\section{Introduction}

Multilevel inverters (MLIs) play a key role in the advancement of electronic technology for the high-power industrial applications. The MLIs offer better waveform quality of output voltage with reduced voltage rating of power semiconductor switches $[1,2]$.

Despite the wide acceptance of MLI, it is still a concern of research, mainly for the improvement in modulation methods $[3,4]$. The selective harmonic elimination pulsewidth modulation (SHE-PWM) method eliminates certain low-order harmonics, offering low switching frequency. It is based on the solution of a group of simultaneous transcendental equations [5]. Initially, the numerical approach was applied to solve SHE equations. Its effectiveness highly depends on the prediction of the proper initial values [6]. Optimization approach such as particle swarm optimization algorithm finds SHE solution by optimizing fitness function [7]. An algebraic method has been investigated combining the symmetrical polynomial and the Groebner bases, which solves the SHE equations reducing the degree of polynomials [8]. The number of harmonics eliminated by conventional SHE methods is restricted by the number of switching angles and dc sources. Real-time implementation is also challenging due to computational burden involved in solving SHE equations.

The SHE-PWM can be implemented with symmetrical and asymmetrical cascaded H-bridge (CHB) MLIs. Symmetrical CHB MLI have all dc sources with the same voltage, while voltages of $\mathrm{dc}$ sources are different in asymmetrical CHB MLI to provide higher levels in output.

*For correspondence
In SHE-PWM-controlled asymmetrical CHB MLI, the voltage ratings of the semiconductor devices are different in each H-bridge. Therefore, the voltage margin available between device voltage ratings and dc voltage source is also different. This limits its operation in abnormal conditions such as overvoltage spikes [2].

Balancing of the volt-second area-based method is proposed, in which voltage of dc-link is considered as a degree of freedom [9]. Its implementation requires dc-dc converter at the input of the each H-bridge of CHB inverter to regulate the input voltage, which results into poor dynamic response. In this letter, an SHE-PWM method is proposed working with constant voltages of dc sources. The concept of volt-second area balancing is applied to estimate the voltage ratings of the dc sources, which provides different voltage ratings of each dc source. Gauss formula of numerical integration is used to calculate the volt-second area. The voltage at inverter output is regulated by uniformly varying the width of switching notch, calculated using univariate equations. It finds switching angles in real time, even for the inverter with a higher number of levels where conventional methods fail. The presented method eliminates a considerable number of harmonics from inverter output voltage.

\section{Proposed SHE-PWM method}

This method is proposed for a CHB inverter. Figure 1 shows an $N$-cell CHB inverter. Each cell is a H-bridge inverter fed by isolated dc voltage sources having different voltage ratings. For the magnitude of individual dc source as $E_{d c i}$, a $\mathrm{H}$-bridge can generate $+E_{d c i}, 0$ and $-E_{d c i}$ voltage 


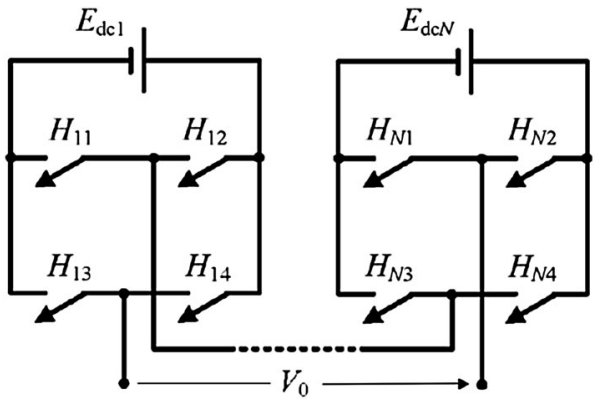

Figure 1. $\mathrm{CHB}$ inverter.

at its output by triggering power switches $H_{i 1}, H_{i 2}, H_{i 3}$ and $H_{i 4}(i=1,2, \ldots, N)$. In the proposed SHE-PWM method, CHB inverter generates $L=2 N$ output levels as zero voltage level is ignored. Output level voltage $\left(E_{\mathrm{s} i}\right)$ and voltage of dc sources $\left(E_{\mathrm{d} c i}\right)$ can be expressed as

$$
\begin{gathered}
E_{\mathrm{s} i}=\sum_{j=1}^{i} E_{\mathrm{d} c j}, \quad i=1,2, \ldots, N . \\
\left\{\begin{array}{l}
E_{\mathrm{d} c 1}=E_{\mathrm{s} 1} \\
E_{\mathrm{d} c i}=E_{\mathrm{s} i}-E_{\mathrm{s}(i-1)}, \quad i=2,3 \ldots, N .
\end{array}\right.
\end{gathered}
$$

Figure 2a shows sinusoidal reference signal having a peak amplitude of $V_{r m}$ and inverter output voltage. Using

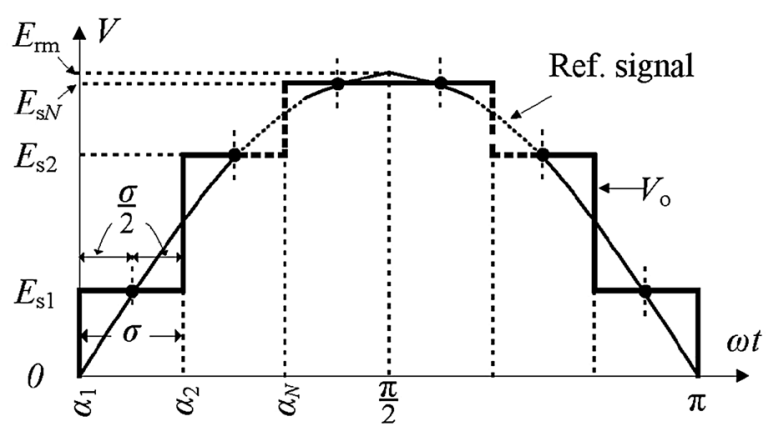

(a)

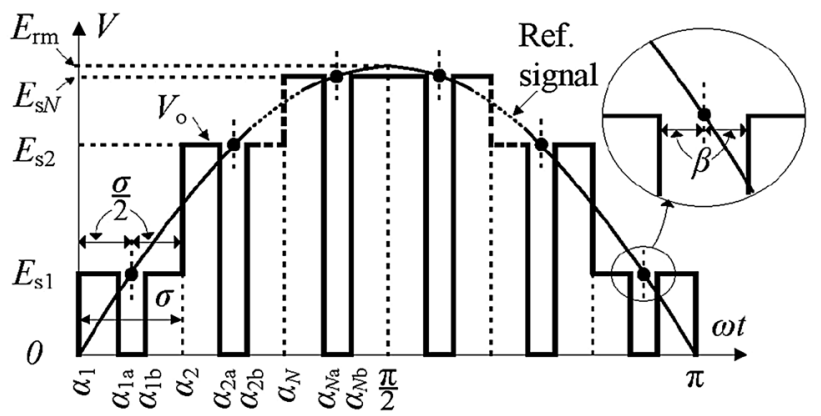

(b)

Figure 2. Working principle of proposed SHE-PWM method: (a) selection of voltage rating of dc sources and (b) the output voltage control by adding switching notch.
Gauss formula of numerical integration [10], the switching angles and voltage of dc sources are selected such that it can balance the area under reference signal and output voltage as follows.

$$
\int_{0}^{\pi / 2} E_{\mathrm{r} m} \sin (y) d y \approx \sum_{i=1}^{L / 2} \sigma E_{\mathrm{s} i}
$$

where

$$
\begin{gathered}
\sigma=\frac{\pi}{L} \\
E_{\mathrm{s} i}=E_{\mathrm{r} m} \sin \left((2 i-1) \frac{\sigma}{2}\right) .
\end{gathered}
$$

Substituting (5) into (2), selection of voltage rating of the dc sources can be done.

To control the output voltage, a switching notch having width of $2 \beta$ is inserted at mid-point of each rectangle in the waveform shown in figure $2 \mathrm{a}$. Resulted waveform of output is presented in figure $2 \mathrm{~b}$. The output voltage can be controlled by varying the value of $\beta$. Considering these notches, switching angles are defined as

$$
\left\{\begin{array}{l}
\alpha_{i}=(i-1) \sigma \\
\alpha_{i \mathrm{a}}=(2 i-1) \frac{\sigma}{2}-\beta \\
\alpha_{i \mathrm{~b}}=(2 i-1) \frac{\sigma}{2}+\beta, \quad i=1,2, \ldots, N .
\end{array}\right.
$$

As per figure $2 \mathrm{~b}$, switching frequency of power switches in top $\mathrm{H}$-bridge is minimum and in bottom $\mathrm{H}$-bridge it is maximum. Total switching frequency of CHB MLI is denoted as $f_{s w}$, which can be expressed as

$$
f_{s w}=25 L(L+4) \text {. }
$$

The synthesized waveform of output voltage can be represented in the Fourier form as follows [11]:

$$
V_{n}=\frac{4}{n \pi} \sum_{i=1}^{N}\left\{E_{\mathrm{d} c i} \cos \left(n \alpha_{i}\right)-E_{\mathrm{s} i}\left(\cos \left(n \alpha_{i a}\right)-\cos \left(n \alpha_{i b}\right)\right)\right\}
$$

Using (2) and (5), it can be represented as

$$
\begin{aligned}
V_{n}= & \frac{4 E_{\mathrm{r} m}}{n \pi}\left[\sin \left(\frac{\sigma}{2}\right)+\sum_{i=2}^{N}\left\{\left\{\sin \left((2 i-1) \frac{\sigma}{2}\right)-\sin \left((2 i-3) \frac{\sigma}{2}\right)\right\}\right.\right. \\
& \times \cos ((i-1) n \sigma)\}-\sum_{i=1}^{N}\left\{\left\{\cos \left(n(2 i-1) \frac{\sigma}{2}-n \beta\right)\right.\right. \\
& \left.\left.\left.-\cos \left(n(2 i-1) \frac{\sigma}{2}+n \beta\right)\right\} \sin \left((2 i-1) \frac{\sigma}{2}\right)\right\}\right] .
\end{aligned}
$$

It can be simplified as 


$$
\begin{aligned}
V_{n}= & \frac{4 E_{\mathrm{r} m}}{n \pi}\left[\operatorname { s i n } ( \frac { \pi } { 2 L } ) \left\{\frac{\sin \left((n+1) \frac{\pi}{4}\right)}{\sin \left((n+1) \frac{\pi}{2 L}\right)} \cos \left((n+1)\left(\frac{\pi}{4}-\frac{\pi}{2 L}\right)\right)\right.\right. \\
& \left.+\frac{\sin \left((n-1) \frac{\pi}{4}\right)}{\sin \left((n-1) \frac{\pi}{2 L}\right)} \cos \left((n-1)\left(\frac{\pi}{4}-\frac{\pi}{2 L}\right)\right)-1\right\}-\sin (n \beta) \\
& \times\left\{\frac{\sin \left((L+2)(n-1) \frac{\pi}{4 L}\right)}{\sin \left((n-1) \frac{\pi}{2 L}\right)} \cos \left((n-1)(L-2)\left(\frac{\pi}{4 L}\right)\right)\right. \\
& -\frac{\sin \left((L+2)(n+1) \frac{\pi}{4 L}\right)}{\sin \left((n+1) \frac{\pi}{2 L}\right)} \cos \left((n+1)(L+2)\left(\frac{\pi}{4 L}\right)\right. \\
& \left.-\sin \left(\frac{\pi}{2 L}\right) \sin \left(\frac{n \pi}{2 L}\right)\right\} .
\end{aligned}
$$

The summation of cosine series results as follows.

$$
\sum_{i=0}^{m-1} \cos (p+i q)=\frac{\sin (m q / 2)}{\sin (q / 2)} \cos \left(\frac{2 p+(m-1) q}{2}\right) .
$$

Considering (11) and L'Hospital's rule, Eq. (10) can be further simplified as

$$
\left\{\begin{aligned}
V_{n}=0 \quad \text { for } \quad n \neq 2 k L \pm 1 \\
=R \quad \text { for } \quad n=1,2 k L \pm 1 \quad(k=1,2, \ldots)
\end{aligned}\right.
$$

where $R=f(n, L, \beta) \neq 0$.

It proves that the present harmonics in the output voltage are $2 k L \pm 1$ for $0 \leqslant \beta \leqslant \sigma / 2$. As per (10), fundamental output voltage $\left(V_{1}\right)$ is a function of only $\beta$ for fixed number of inverter levels. Therefore, fundamental output voltage can be controlled by varying value of $\beta$.

\section{Implementation}

Implementation of the proposed method is accomplished in two steps: (1) selection of voltage rating of dc-links and (2) real-time calculation of switching angles.

The selection of voltage ratings of dc-links are done using Volt_selection() algorithm. Its pseudo-code is presented as algorithm 1. Real-time calculation of switching angles is implemented with Angle_calc() algorithm. Algorithm 2 shows the pseudo-code of the Angle_calc() algorithm.

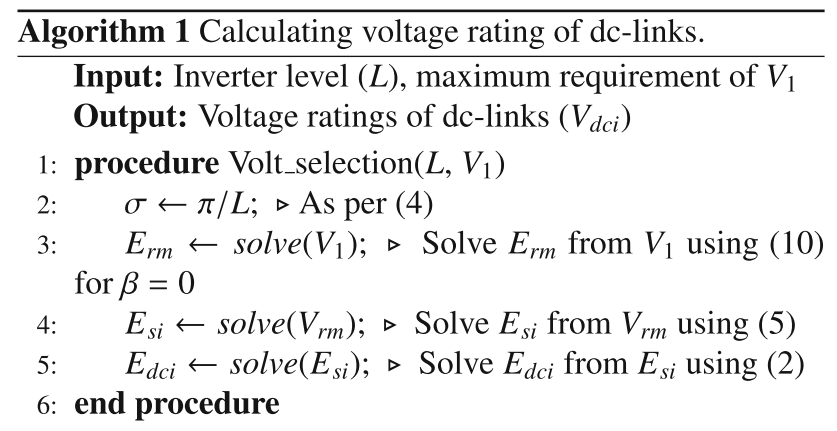

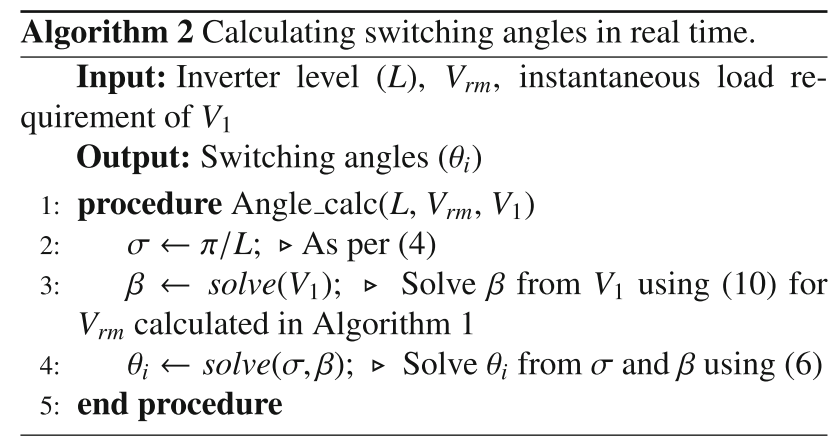

Using Algorithms 1 and 2, a sample calculation is presented for a six-level inverter as follows. For six-level inverter, $\sigma=\pi / 6$. The value of $E_{\mathrm{r} m}=101.15 \mathrm{~V}$ for $\beta=0$ rad considering maximum required $V_{1}=100 \mathrm{~V}$. Accordingly, $E_{\mathrm{s} 1}, E_{\mathrm{s} 2}$ and $E_{\mathrm{s} 3}$ are calculated, respectively, as 26.18, 71.53 and $97.71 \mathrm{~V}$. Using these, selections of voltage ratings of dc sources $\left(E_{\mathrm{d} c 1}, E_{\mathrm{d} c 2}\right.$ and $\left.E_{\mathrm{d} c} 3\right)$ are, respectively, $26.18,45.35$ and $26.18 \mathrm{~V}$.

For $V_{1}=100 \mathrm{~V}, \beta=0 \mathrm{rad}$ and switching angles $(\alpha)$ are $0, \pi / 6$ and $\pi / 3$ rad. Similarly, for $V_{1}=95 \mathrm{~V}, \beta=0.0129$ rad and switching angles are 0, 0.249, 0.275, 0.524, 0.772, $0.798,1.047,1.296$ and 1.322 rad.

\section{Simulation and experimental results}

Simulation and experimental studies have been carried out for the six-level CHB inverter, using system parameters calculated in the previous section.

Figure 3 shows simulation results of six-level inverter acquired in MATLAB/Simulink environment. The value of $V_{1}$ is $100 \mathrm{~V}$ for $\beta=0 \mathrm{rad}$ and it reduces to $95 \mathrm{~V}$ for $\beta=0.0129 \mathrm{rad}$, maintaining voltage of $\mathrm{dc}$ sources constant. The harmonic pattern $2 k L \pm 1$ is not affected while creating switching notch for voltage control. In the THD spectrum, present harmonics are 11-, 13-, 23-, 25-, 35-, 37-, 47- and 49th, up to 50th harmonics, which correspond well with (12). THD of output voltage is $14.17 \%$ for $\beta=$ $0 \mathrm{rad}$ and $20.43 \%$ for $\beta=0.0129 \mathrm{rad}$. As per (7), switching frequency is $750 \mathrm{~Hz}$ for six-level inverter. All harmonics are eliminated up to 50th harmonic from the THD spectrum of the output voltage of the 26-level inverter, which works with switching frequency of $19.5 \mathrm{kHz}$.

An experimental study has been carried out for six-level inverter implementing control scheme in an ARM CortexM4 STM32F407VG microcontroller. The six-level inverter is implemented using FGA25N120 IGBTs from Fairchild Semiconductor. The associated driver circuit is designed using a 1ED020I12F2 chip from Infineon Technologies. Figure 4 shows output voltage and its THD spectrum for $\beta=0.0129 \mathrm{rad}$. The measured value of $V_{1}$ is 87.96 $(=\sqrt{2} \times 62.2) \mathrm{V}$ and THD of output voltage is $20.50 \%$. 


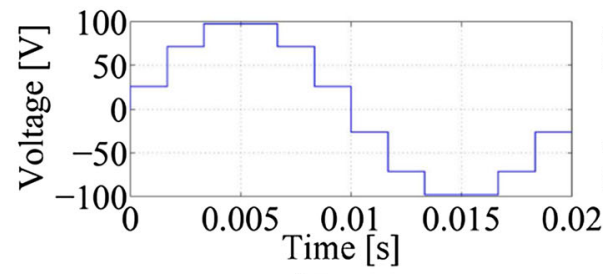

(a)

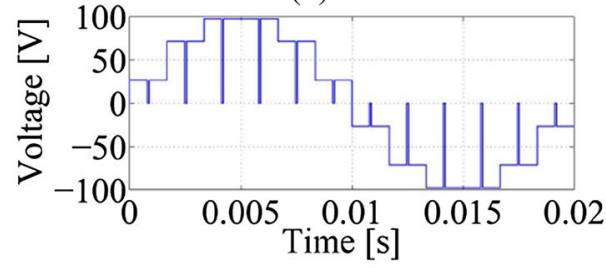

(c)

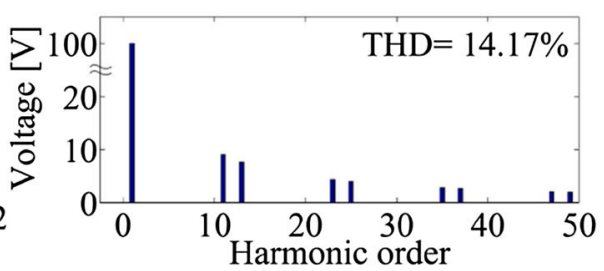

(b)

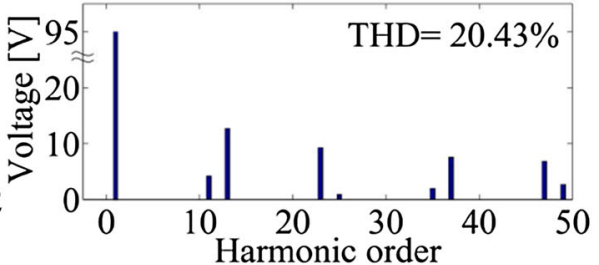

(d)

Figure 3. Simulation results of proposed method with six-level MLI: (a) output voltage, (b) its THD spectrum for $\beta=0$ rad, (c) output voltage and (d) its THD spectrum for $\beta=0.0129 \mathrm{rad}$.

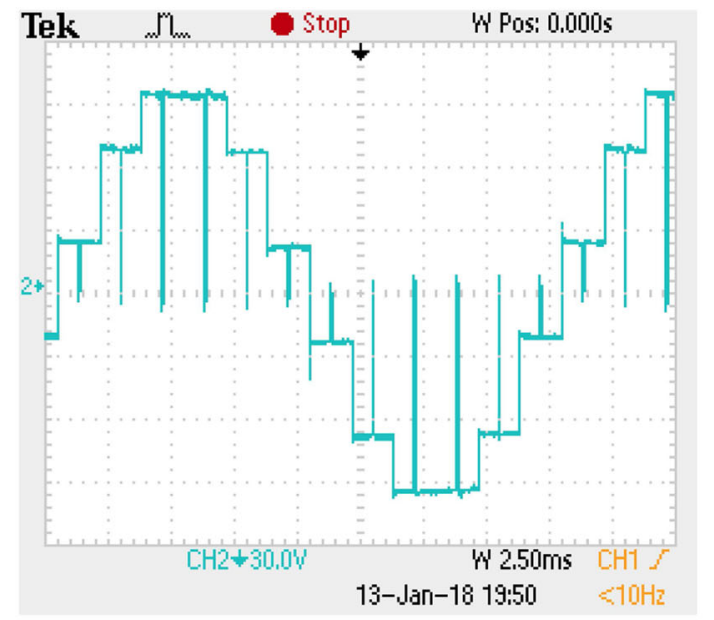

(a)

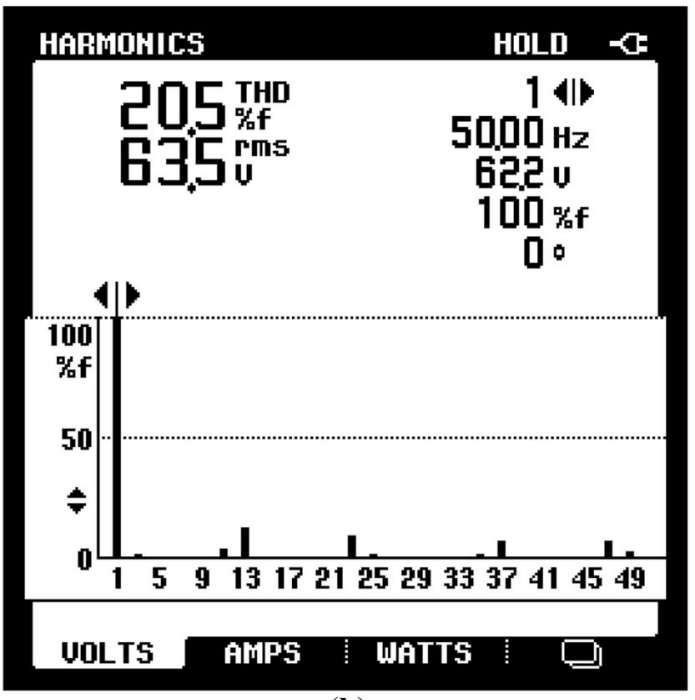

(b)

Figure 4. Experimental result of six-level MLI for $\beta=0.0129$ rad: (a) output voltage and (b) THD spectrum of output voltage.

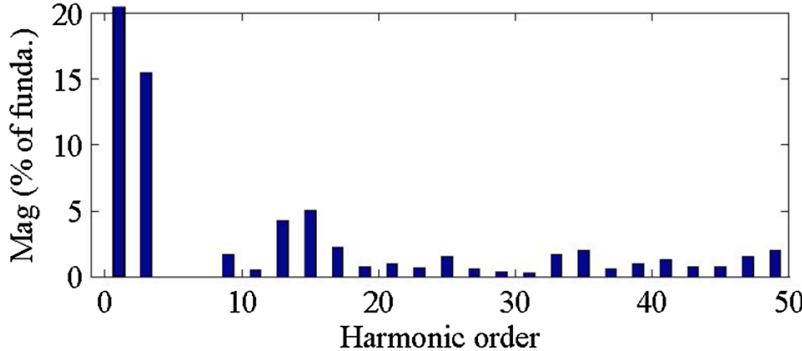

(a)

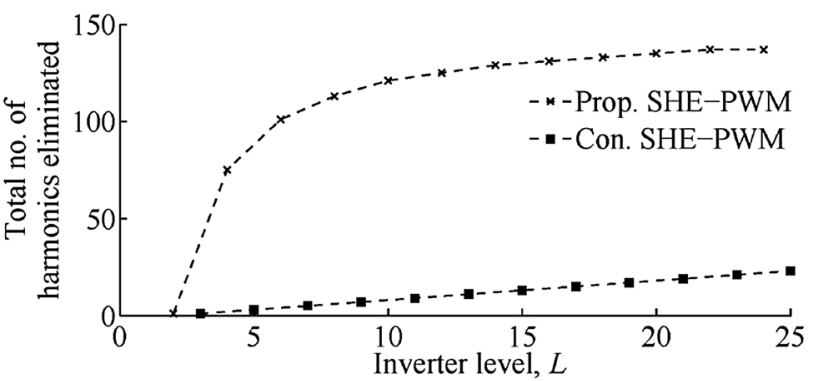

(b)

Figure 5. Comparative study: (a) THD spectrum of conventional SHE-PWM method and (b) number of odd harmonics eliminated up to 300 th order harmonic.

These results have good accordance with the simulation results.

A comparative study has been done with the conventional SHE method [11]. Conventional SHE-PWM method eliminates totally $(L-3) / 2$ number of harmonics from the output voltage of inverter. Figure 5a shows harmonics spectrum of conventional SHE-PWM method with sevenlevel CHB MLI. It shows that the conventional method can eliminate only two harmonics, while the proposed SHE- 
PWM method eliminates a large number of harmonics except $2 k L \pm 1$ order harmonics. Figure $5 \mathrm{~b}$ shows total number of harmonics eliminated up to 300th order harmonics. It depicts that the proposed method eliminates a significant number of harmonics compared with the conventional SHE-PWM method.

\section{Conclusions}

In this article, a univariate-equation-based SHE-PWM method is proposed. Due to reduced mathematical burden, this method can be implemented in real time for a large number of inverter levels using general-purpose microcontrollers. While controlling the output voltage by varying width of switching notch with fixed voltage of dc sources, only magnitude of harmonics changes without affecting the harmonic pattern. This method eliminates a large number of harmonics compared with the conventional SHE-PWM methods. In the six-level inverter, switching frequency is $750 \mathrm{~Hz}$ and only 11-, 13-, 23-, 25-, 35-, 37-, 47- and 49th harmonics are present up to 50th harmonics. A 26-level inverter operates at $19.5 \mathrm{kHz}$ switching frequency, in which all harmonics are eliminated up to 50th harmonic from the THD spectrum of the output voltage.

\section{References}

[1] Sarkar I and Fernandes B G 2017 A nine-level hybrid symmetric cascaded multilevel converter for induction motor drive. Sadhana 42: 1389-1400
[2] Kouro S, Malinowski M, Gopakumar K, Pou J, Franquelo L G, Wu B, Rodriguez J, Perez M A and Leon J I 2010 Recent advances and industrial applications of multilevel converters. IEEE Trans. Ind. Electron. 57: 2553-2580

[3] Edpuganti A and Rathore A K 2015 A survey of low switching frequency modulation techniques for medium-voltage multilevel converters. IEEE Trans. Ind. Appl. 51: 4212-4228

[4] Sangeetha S and Jeevananthan S 2015 Influence of crossover methods used by genetic algorithm-based heuristic to solve the selective harmonic equations (SHE) in multi-level voltage source inverter. Sadhana 40: 2389-2410

[5] Dahidah M S A 2010 Selective harmonic elimination nonsymmetrical bipolar pulse width modulation technique: analysis and experimental verification. J. Circuits Syst. Comput. 19: 719-731

[6] Patel H S and Hoft R G 1973 Generalized techniques of harmonic elimination and voltage control in thyristor inverters: part I-harmonic elimination. IEEE Trans. Ind. Appl. 9: 310-317

[7] Etesami M, Ghasemi N, Vilathgamuwa D M and Malan W L 2017 Particle swarm optimisation-based modified SHE method for cascaded $\mathrm{H}$-bridge multilevel inverters. IET Power Electron 10: 18-28

[8] Yang K, Zhang Q, Yuan R, Yu W, Yuan J and Wang J 2016 Selective harmonic elimination with Groebner bases and symmetric polynomials. IEEE Trans. Power Electron. 31: 2742-2752

[9] Kamani P L and Mulla M A 2018 Middle-level SHE pulseamplitude modulation for cascaded multilevel inverters. IEEE Trans. Ind. Electron. 65: 2828-2833

[10] Suli E and Mayers D F 2003 An introduction to numerical analysis. Cambridge: Cambridge University Press

[11] Dahidah M S A, Konstantinou G and Agelidis V G 2015 A review of multilevel selective harmonic elimination PWM: formulations, solving algorithms, implementation and applications. IEEE Trans. Power Electron. 30: 4091-4106 